\title{
Prevalência de pensamentos e comportamentos suicidas e associação com a insatisfação corporal em adolescentes
}

\author{
Prevalence of suicidal thoughts and behaviors and its \\ association with body dissatisfaction in adolescents \\ Gaia Salvador Claumann', André de Araújo Pinto', Diego Augusto Santos Silva², Andreia Pelegrini
}

\section{RESUMO}

Objetivo: Estimar a prevalência de pensamentos e comportamentos suicidas e a associação com a insatisfação corporal em adolescentes. Métodos: Participaram 1.090 adolescentes (501 do sexo masculino e 589 do sexo feminino), com média de 16,2 (1,1) anos de idade, estudantes do ensino médio em São José-SC. Por meio de questionário autoadministrado, os adolescentes responderam a questões sociodemográficas (sexo, idade) e sobre maturação sexual, insatisfação corporal (escala de silhuetas) e pensamentos e comportamentos suicidas (ideação, planejamento e tentativa de suicídio), e tiveram as medidas de peso corporal e altura aferidas para cálculo do índice de massa corporal (IMC = peso corporal dividido pela altura ao quadrado). Empregou-se a regressão logística binária para análise dos dados. Resultados: $O$ sexo feminino apresentou maiores prevalências de pensamento, planejamento e tentativa de suicídio comparado ao masculino. Os adolescentes insatisfeitos pelo excesso de peso e pela magreza apresentaram maior chance de terem pensado e planejado suicídio. Não foram encontradas associações entre tentativa de suicídio e insatisfação corporal. Conclusões: $O$ sexo feminino apresentou maiores prevalências de pensamentos e comportamentos suicidas do que o masculino. Independentemente do sexo, idade, IMC e maturação sexual, os adolescentes insatisfeitos com o corpo (pelo excesso de peso e pela magreza) estiveram mais suscetíveis à ideação suicida e ao planejamento de suicídio, comparados aos satisfeitos. Por serem adolescentes em idade escolar, a escola pode ser um agente de discussão sobre a temática, auxiliando na prevenção da insatisfação corporal e desfechos suicidas. Outros profissionais envolvidos com essa população e os familiares precisam estar atentos a essas questões.

\section{Palavras-chave}

Suicídio, saúde do adolescente, imagem corporal.

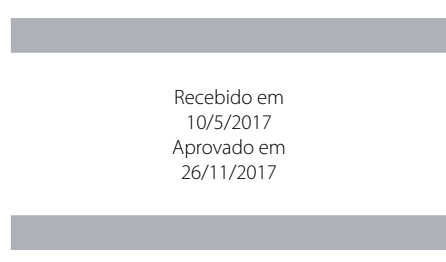

DOI: 10.1590/0047-2085000000177
1 Universidade do Estado de Santa Catarina (UFSC), Centro de Ciências da Saúde, Programa de Pós-graduação em Ciências do Movimento Humano, Florianópolis, SC, Brasil.

2 Universidade Federal de Santa Catarina (UFSC), Centro de Desportos, Programa de Pós-graduação em Educação Física, Florianópolis, SC, Brasil.
Endereço para correspondência: Andreia Pelegrini Universidade do Estado de Santa Catarina (UFSC), Centro de Ciências da Saúde e do Esporte,

Grupo de Estudo e Pesquisa em Cineantropometria (GEPECIN)

Rua Pascoal Simone, 358, Coqueiros

88080-350 - Florianópolis, SC, Brasil

Telefone: (48) 3664-8695

E-mail: andreia.pelegrini@udesc.br 


\section{Keywords}

Suicide, adolescent health, body image.

\section{ABSTRACT}

Objective: To estimate the prevalence of suicidal thoughts and behaviors and its association with body image dissatisfaction in adolescents. Methods: Participants were 1,090 adolescents (501 male and 589 female), with mean age of 16.2 (1.1) years, who were high school students in Sao Jose-SC. In a self-administered questionnaire, adolescents answered sociodemographic questions (sex, age), sexual maturation, body dissatisfaction (figure rating scale), suicidal thoughts and behaviors (ideation, planning and attempts), and had body weight and height measured to calculate the body mass index (BMI = body weight divided by squared height). Binary logistic regression was used to data analysis. Results: Greater prevalence of ideation, planning and suicidal attempts was found among female than male. Adolescents who were dissatisfied by thinness and by overweight were more likely to ideation and planning suicide. No associations between suicidal attempts and body image dissatisfaction were found. Conclusions: Females had higher prevalence of ideation, planning and attempt than males. Regardless of sex, age, BMI and sexual maturation, the adolescents who were dissatisfied with their body (both by thinness and by overweight) were more likely to ideation and planning the suicide, compared to those who were satisfied. Giving that these adolescents are in school age, the school can be an agent of discussion on the subject, assisting to prevent body dissatisfaction and suicidal outcomes. Other professionals who work with adolescents and relatives need to pay attention to these issues.

\section{INTRODUÇÃO}

Mundialmente, estima-se que a cada ano mais de 800.000 pessoas morrem por suicídio - uma pessoa a cada 40 segundos ${ }^{1}$. No ano de 2012, o suicídio foi a $15^{\text {a }}$ principal causa de morte no mundo, contribuindo para 1,4\% de todos os óbitos ocorridos'. Os dados sobre suicídio são ainda mais alarmantes quando se considera a população jovem. Entre indivíduos com idades de 15 a 29 anos, em 2012, o suicídio contribuiu para 8,5\% do total de óbitos, tornando-se a segunda principal causa de morte nessa população, mundialmente'. Em alguns países, porém, o suicídio foi a principal causa de morte, no mesmo período, entre indivíduos dessa faixa etária, contribuindo, por exemplo, para $17,6 \%$ e $16,6 \%$ do total de óbitos, em países de alta renda e de baixa/média renda, respectivamente, do Sudeste da Ásia'.

No Brasil, no período de 2011 a 2016, foram registrados no Sistema de Informação de Agravos de Notificação (Sinan) 48.204 casos de tentativas de suicídio, os quais ocorreram predominantemente nas regiões Sudeste e Sul do país. Grande parte dessas tentativas foi realizada por adolescentes com idades de 10 a 19 anos, sendo $24,1 \%$ pelo sexo feminino e $17,2 \%$ pelo sexo masculino ${ }^{2}$. Ainda de acordo com registros do Sinan, nos anos de 2011 a 2015 ocorreram 55.649 óbitos por suicídio no Brasil, e o risco aumentou ao longo do período. Essas mortes por suicídio ocorreram com maior frequência nos estados do Rio Grande do Sul, Santa Catarina e Mato Grosso do Sul, respectivamente 2 .

Diante desse cenário, o suicídio é, notavelmente, um dos principais problemas de saúde pública em todo o mundo e resulta em prejuízos econômicos, sociais e psicológicos para indivíduos, famílias, comunidades e países inteiros'. Isso tem motivado pesquisadores de diferentes áreas do conhecimento a investigarem os pensamentos e comportamentos suicidas como um dos primeiros prognósticos do suicídio consumado ${ }^{3-6}$, de modo a possibilitar o desenvolvimento de estratégias para a prevenção deles, minimizando-se as mortes por essa causa. Os pensamentos e comportamentos suicidas, segundo Franklin et al. ${ }^{7}$, compreendem a ideação (pensamentos sobre matar a si mesmo), o planejamento (considerar um método específico por meio do qual tem a intenção de se matar) e a tentativa (envolver-se em comportamentos que ocasionam lesões autoprovocadas, nos quais há alguma intenção de morrer em decorrência deles). Porém, assim como a maioria de outros constructos psicológicos, não há conceitos universalmente aceitos das formas específicas de pensamentos e comportamentos suicidas? ${ }^{7}$.

Os pensamentos e comportamentos suicidas são fenômenos complexos e influenciados por inúmeros fatores que interagem entre si, como pessoais, sociais, psicológicos, culturais, biológicos e ambientais'. Assim, nenhum fator isoladamente é suficiente para explicar por que uma pessoa morre por suicídio'. Apesar disso, alguns fatores parecem ser preditores mais fortes dos diferentes desfechos relacionados ao suicídio, conforme apontaram os resultados de uma revisão sistemática com metanálise que incluiu estudos longitudinais publicados ao longo de 50 anos $^{7}$. Independentemente da faixa etária da população investigada, fatores como tentativas prévias de suicídio, lesões prévias autoprovocadas sem a intenção de se matar, hospitalizações psiquiátricas prévias, 
ansiedade, depressão e desespero, além de eventos de vida estressantes, tiveram destaque?.

Dessa forma, a adolescência pode ser considerada um evento de vida estressante devido às inúmeras e intensas mudanças físicas e psicológicas ocorridas nesse período, dentre as quais se destacam as mudanças atreladas à puberdade em diferentes aspectos e no formato do corpo que muitas vezes deixam os jovens desconfortáveis e confusos com sua imagem corporal, tornando-se um desafio para eles ${ }^{8}$. As dificuldades dos adolescentes em lidar com a imagem corporal estão relacionadas a sintomas de baixa autoestima, ansiedade e depressão9-11, os quais, por sua vez, podem predispor a inúmeros comportamentos que põem em risco a vida do indivíduo e, especificamente, a pensamentos e comportamentos suicidas ${ }^{12-15}$, o que faz com que a imagem corporal constitua um potencial fator de risco para o suicídio nessa fase da vida.

Nesse sentido, estudos verificaram que diferentes componentes da imagem corporal (por exemplo, afetivo, cognitivo e comportamental) estão relacionados às lesões não suicidas autoprovocadas por adolescentes e jovens adultos ${ }^{16,17}$. Assim, seria adequado supor que o componente da insatisfação com a imagem corporal também teria relações com esses e outros comportamentos mais graves. No entanto, pesquisas que investigaram diretamente a relação entre a insatisfação corporal e diferentes desfechos relacionados ao suicídio ${ }^{18-20}$ produziram resultados distintos, que em alguns casos revelaram associações apenas com uma das formas de pensamentos e comportamentos suicidas (ideação, planejamento ou tentativa) e em outros não apontaram associação alguma.

Apesar da falta de consenso nos estudos, o fato de alguns deles demonstrarem que há relação entre insatisfação com a imagem corporal e ideação, planejamento e tentativas suicidas, sugere a necessidade de maior aprofundamento sobre essa questão e atenção dos pesquisadores, profissionais envolvidos com adolescentes e órgãos de saúde pública. Dessa forma, percebe-se a urgência de ampliar o conhecimento sobre esse assunto, buscando compreender, principalmente, a realidade em um contexto nacional para que possam ser elaboradas estratégias adequadas que auxiliem na prevenção dos problemas de imagem corporal e dos pensamentos e comportamentos suicidas. Portanto, o objetivo do presente estudo foi verificar a prevalência de pensamentos e comportamentos suicidas e a associação com a insatisfação com a imagem corporal em adolescentes.

\section{MÉTODOS}

\section{População e amostra}

Este estudo epidemiológico, transversal, de base escolar, está vinculado ao macroprojeto "Guia Brasileiro de Avaliação da Aptidão Física Relacionada à Saúde e Hábitos de Vida - Etapa l", o qual foi aprovado pelo Comitê de Ética em Pesquisas com Seres Humanos da instituição de origem (Protocolo no 746.536/2014).

A população desta pesquisa era formada por 5.182 escolares de 14 a 19 anos de idade, estudantes do ensino médio de escolas públicas estaduais da cidade de São José-SC, distribuídos em 11 escolas elegíveis e 170 turmas do ensino médio. O processo amostral foi determinado em dois estágios: 1) estratificado por escolas públicas estaduais de ensino médio ( $n=11)$; 2 ) conglomerado de turmas, considerando turno de estudo e série de ensino ( $n=170$ turmas). No estágio dois, foram convidados a participar do estudo todos os estudantes do ensino médio que estavam presentes em sala de aula nos dias das coletas de dados. A amostra probabilística foi formada por 1.132 estudantes. Detalhes sobre as estimativas para cálculo do tamanho de amostra e todo o processo de amostragem (critérios de inclusão, exclusão, elegibilidade) podem ser consultados na literatura ${ }^{21}$.

\section{Procedimentos e variáveis do estudo}

As coletas de dados foram realizadas após a autorização da Secretaria de Estado da Educação, em dias e horários combinados com os diretores e professores das escolas que aceitaram participar da pesquisa. Os estudantes das turmas sorteadas foram informados sobre os objetivos e a importância da pesquisa e receberam o Termo de Consentimento Livre e Esclarecido (TCLE) para ser assinado por um responsável, autorizando-os a fazer parte dela. Após o recolhimento do TCLE, os estudantes responderam a um questionário autoaplicável e assinaram ao Termo de Assentimento.

Os pensamentos e comportamentos suicidas (ideação, planejamento e tentativa de suicídio) constituíram a variável dependente do presente estudo, os quais foram coletados por meio de questões extraídas do questionário Youth Risk Behavior Survey (YRBS), traduzido e validado para a população brasileira, apresentando valores do Índice de Concordância Kappa satisfatórios (entre $76,8 \%$ e $86,0 \%$ ) $^{22}$. Os adolescentes foram instruídos a considerarem os últimos 12 meses para responder às seguintes questões: 1) "Você em algum momento pensou seriamente em cometer suicídio (se matar)?" (Ideação suicida); 2) "Você já planejou como cometer um suicídio?" (Planejamento suicida); e 3) "Quantas vezes você efetivamente tentou suicídio?" (Tentativa de suicídio). As questões 1 e 2 tinham como opções de resposta "sim" e "não". A questão 3 apresentava cinco opções de resposta (nenhuma vez, uma vez, duas ou três vezes, quatro ou cinco vezes e seis vezes ou mais). Para o presente estudo, nessa última questão, a resposta "nenhuma vez" foi considerada como "não" tentou suicídio e todas as demais foram consideradas "sim", tentou suicídio.

A variável independente foi a insatisfação com a imagem corporal, avaliada por meio de uma escala de nove silhuetas para cada sexO ${ }^{23} \mathrm{com}$ validade para amostras brasileiras ${ }^{24}$. Os adolescentes indicaram o número da silhueta (de 1 a 9 , sendo a silhueta de número 1 a de menores dimensões e 
a de número 9 a de maiores dimensões corporais) que meIhor representava sua forma física "atual" e posteriormente aquela que gostariam de se parecer, a "ideal". Para determinar a insatisfação ou a satisfação com a imagem corporal, a silhueta "ideal" foi subtraída da "atual", sendo classificados como satisfeitos aqueles em que o resultado da subtração foi igual a "zero", insatisfeitos pela magreza quando o resultado foi "menor do que zero" e insatisfeitos pelo excesso de peso quando o resultado foi "maior do que zero".

Além disso, no presente estudo, as variáveis sexo (feminino e masculino), idade em anos completos $(14,15,16,17$, 18 e 19), índice de massa corporal (IMC) e maturação sexual foram utilizadas para ajustar as análises de associação entre pensamentos e comportamentos suicidas e insatisfação com a imagem corporal.

Para o cálculo do IMC, as medidas de peso corporal e altura foram aferidas. O peso corporal foi aferido por meio de balança digital da marca G Tech Pro ${ }^{\circledR}$ (Pacific Palisades, USA), com capacidade de até $150 \mathrm{~kg}$ e resolução de $100 \mathrm{~g}$. A altura foi aferida por meio de estadiômetro da marca Sanny ${ }^{\circledast}$ (São Paulo, Brasil), com resolução de $0,1 \mathrm{~cm}$. Para a realização de ambas as medidas, foram seguidos os procedimentos da International Standards For Anthropometry Assessment (ISAK) ${ }^{25}$.

A maturação sexual foi obtida por meio de autorrelato. Os adolescentes indicaram o estágio de desenvolvimento de pelos pubianos que melhor correspondia ao seu corpo a partir de figuras adaptadas das fotografias de Tanner (1962)26. Os cinco estágios apresentados foram classificados em pré-púberes (estágio 1), púberes (estágios 2, 3 e 4) e pós-púberes (estágio 5). Os participantes responderam a essa questão individualmente, somente após explicação do pesquisador responsável sobre o instrumento.

\section{Análise estatística}

Os dados foram analisados por meio de estatística descritiva (média, desvio-padrão e distribuição de frequências) e inferencial. A associação entre cada uma das formas de pensamentos e comportamentos suicidas (ideação, planejamento e tentativas de suicídio) e a insatisfação com a imagem corporal foi testada por meio da regressão logística binária, conduzindo-se análises brutas e ajustadas (por sexo, idade, IMC e maturação sexual), estimando-se a odds ratio (OR) e os respectivos intervalos de confiança de 95\% (IC 95\%). Todas as análises foram realizadas no software IBM SPSS Statistics 20, com nível de significância de 5\%.

\section{RESULTADOS}

Dos 1.132 participantes do macroprojeto, 42 foram excluídos das análises do presente estudo por não terem respondido às questões referentes à ideação suicida. Dessa forma, foram analisados dados de 1.090 adolescentes (54,0\% do sexo feminino e $46,0 \%$ do sexo masculino). As características dos participantes em relação às variáveis investigadas, na amostra total e de acordo com o sexo, são apresentadas na Tabela 1.

Na Figura 1, são apresentadas as prevalências de pensamentos e comportamentos suicidas na amostra total e de acordo com o sexo. O sexo feminino apresentou maiores prevalências de ideação (16,0\%; IC 95\% = 13,9-17,8), planejamento $(12,1 \%$; IC 95\% = 10,2-13,8) e tentativa suicida $(6,8 \%$; IC $95 \%=6,5-7,0)$ quando comparado ao sexo masculino $(11,6 \%$; IC 95\% = 9,7-13,2; 9,0\%; IC 95\% = 8,8-9,2; 4,2\%; IC 95\% =3,9-4,4, respectivamente).

Tabela 1. Características dos participantes em relação às variáveis investigadas, na amostra total e de acordo com o sexo - São José, SC, Brasil, 2014

\begin{tabular}{|c|c|c|c|}
\hline \multirow[b]{2}{*}{ Variáveis } & \multirow[b]{2}{*}{ Total } & \multicolumn{2}{|c|}{ Sexo } \\
\hline & & $\begin{array}{c}\text { Masculino } \\
(\mathrm{n}=501)\end{array}$ & $\begin{array}{l}\text { Feminino } \\
(\mathrm{n}=589)\end{array}$ \\
\hline Idade, $\bar{x}(d p)$ & $16,2(1,1)$ & $16,3(1,2)$ & $16,1(1,1)$ \\
\hline$I M C, \bar{x}(d p)$ & $22,2(3,8)$ & $21,9(3,5)$ & $22,4(4,1)$ \\
\hline \multicolumn{4}{|l|}{ Imagem corporal, n (\%) } \\
\hline Satisfeito & $266(24,4)$ & $151(30,0)$ & $115(19,5)$ \\
\hline Insatisfeito pelo excesso de peso & $434(39,8)$ & $138(27,5)$ & $296(50,3)$ \\
\hline Insatisfeito pela magreza & $390(35,0)$ & $212(42,5)$ & $178(30,2)$ \\
\hline \multicolumn{4}{|l|}{ Maturação sexual, n (\%) } \\
\hline Pré-púbere & $74(6,8)$ & $37(7,4)$ & $37(6,3)$ \\
\hline Púbere & $868(80,1)$ & $368(73,7)$ & $500(85,5)$ \\
\hline Pós-púbere & $142(13,1)$ & $94(18,8)$ & $48(8,2)$ \\
\hline
\end{tabular}

$\overline{\mathrm{x}}$ : média; dp: desvio-padrão; n: frequência absoluta; \%: frequência relativa. 


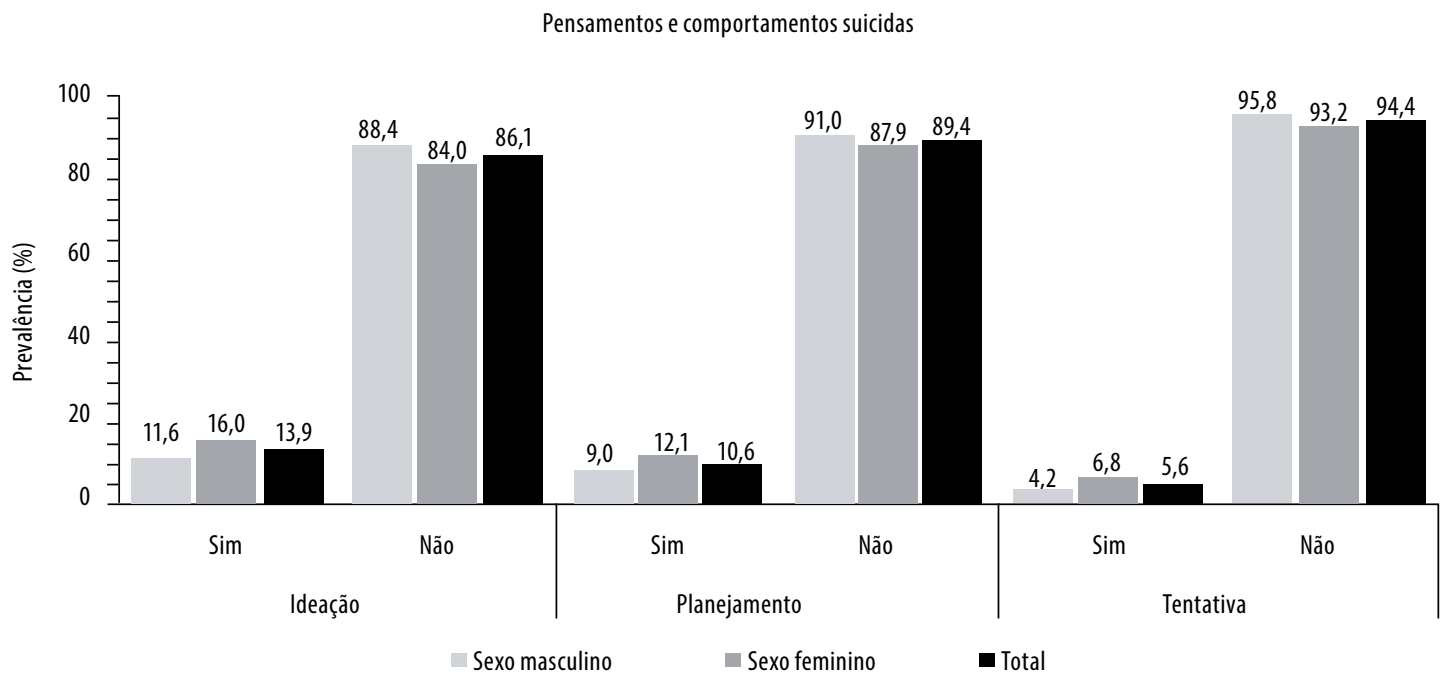

Figura 1. Prevalências de ideação, planejamento e tentativa de suicídio na amostra total e de acordo com o sexo - São José, SC, Brasil.

As análises de associação entre os pensamentos e comportamentos suicidas e a insatisfação com a imagem corporal são apresentadas na Tabela 2. Nas análises brutas, foi observada associação apenas entre ideação suicida e insatisfação pelo excesso de peso. Após o ajuste, verificou-se que os adolescentes insatisfeitos pelo excesso de peso (OR $=2,37 ; \mathrm{IC} 95 \%=1,24-4,51)$ e pela magreza ( $\mathrm{OR}=2,81 ; \mathrm{IC} 95 \%$ $=1,15-5,20)$ tiveram mais chances de apresentar ideação suicida quando comparados aos satisfeitos com a imagem corporal. De forma semelhante, os insatisfeitos pelo excesso de peso $(\mathrm{OR}=2,46$; IC 95\% = 1,21-5,00) e pela magreza (OR $=2,48 ; \mathrm{IC} 95 \%=1,25-4,93)$ apresentaram mais chances de planejar o suicídio comparados aos satisfeitos.

\section{DISCUSSÃO}

O presente estudo investigou a prevalência de pensamentos e comportamentos suicidas em adolescentes de uma cidade do sul do Brasil e auxilia a preencher essa lacuna de informação da literatura nacional. Os resultados apontaram que o sexo feminino apresentou maiores prevalências de pensamentos e comportamentos suicidas (ideação, planejamentos e tentativas) comparado ao sexo masculino. Os adolescentes insatisfeitos (pelo excesso de peso e pela magreza) estiveram mais suscetíveis à ideação e ao planejamento do suicídio em relação aos satisfeitos com a imagem corporal.

Tabela 2. Análise de associação entre planejamentos e comportamentos suicidas (ideação, planejamento e tentativa) e insatisfação com a imagem corporal em adolescentes de São José, SC, 2014

\begin{tabular}{|c|c|c|c|}
\hline Variáveis & $\mathrm{n}(\%)$ & $\begin{array}{l}\text { Análise bruta } \\
\text { OR (IC } 95 \%)\end{array}$ & $\begin{array}{l}\text { Análise ajustada } \\
\text { OR (IC } 95 \%)^{*}\end{array}$ \\
\hline \multicolumn{4}{|l|}{ Ideação } \\
\hline Satisfeitos & $18(6,8)$ & 1 & 1 \\
\hline Insatisfeitos pelo excesso de peso & $75(17,3)$ & $2,54(1,15-5,59)$ & $2,37(1,24-4,51)$ \\
\hline Insatisfeitos pela magreza & $59(15,1)$ & $2,22(0,96-4,39)$ & $2,81(1,15-5,20)$ \\
\hline \multicolumn{4}{|l|}{ Planejamento } \\
\hline Satisfeitos & $14(5,3)$ & 1 & 1 \\
\hline Insatisfeitos pelo excesso de peso & $60(13,8)$ & $2,60(0,94-4,76)$ & $2,46(1,21-5,00)$ \\
\hline Insatisfeitos pela magreza & $42(10,80$ & $2,03(0,55-2,77)$ & $2,48(1,25-4,93)$ \\
\hline \multicolumn{4}{|l|}{ Tentativa } \\
\hline Satisfeitos & $10(3,4)$ & 1 & 1 \\
\hline Insatisfeitos pelo excesso de peso & $26(6,0)$ & $1,76(0,84-3,95)$ & $1,84(0,74-4,52)$ \\
\hline Insatisfeitos pela magreza & $26(6,7)$ & $1,97(0,94-4,43)$ & $2,00(0,86-4,67)$ \\
\hline
\end{tabular}

\%: frequência relativa; OR: odds ratio; IC 95\%: intervalo de confiança de 95\%.

*Análise ajustada por sexo, idade, IMC e maturação sexual. 
Pensamentos e comportamentos suicidas têm sido mais prevalentes em adolescentes do sexo feminino de diversos países $^{5,27-29}$ e do Brasil ${ }^{4,30-32}$. A predisposição do sexo feminino à ideação, planejamento e tentativa de suicídio pode ser reflexo de conflitos internos que incluem depressão, ansiedade e autocobrança mais sensíveis a elas, além de que o sexo feminino tende a se sentir mais pressionado e estressado diante das situações conflituosas da vida em relação ao sexo masculino ${ }^{5}$. Ademais, o sexo masculino, comparado ao feminino, sofre menos por fatores como conflitos domésticos, dificuldades econômicas e escolares, que tendem a contribuir significativamente para os pensamentos e comportamentos suicidas $^{3}$. No entanto, dados nacionais ${ }^{2}$ e internacionais ${ }^{1}$ demonstram que, em todas as faixas etárias, o maior número de óbitos por suicídio ocorre entre o sexo masculino.

Apesar de terem sido verificadas diferenças estatisticamente significativas entre os sexos nas prevalências de pensamentos e comportamentos suicidas, destaca-se que, ao considerar a amostra como um todo, 16,0\% dos adolescentes investigados apresentaram ideação suicida nos últimos 12 meses, 12,1\% planejaram como realizar o suicídio e 6,8\% efetivamente tentaram matar a si mesmos. Todos esses adolescentes merecem maior atenção e devem ser alvos de estratégias de prevenção ao suicídio, principalmente aqueles que já tentaram matar a si mesmos, pois tentativas prévias constituem o principal fator de risco para o óbito por suicídio ${ }^{1,7}$. Ainda, as tentativas de suicídio podem resultar em prejuízos sociais e econômicos para as comunidades devido ao uso dos serviços de saúde para tratar as lesões do indivíduo, bem como, em alguns casos, as incapacidades de longo prazo devidas a elas, além do impacto psicológico e social causado não somente no indivíduo, mas também nas pessoas próximas a ele ${ }^{1}$, o que demonstra a necessidade de acompanhamento e suporte aos adolescentes que apresentam pensamentos e comportamentos suicidas.

Quanto às associações entre pensamentos e comportamentos suicidas e insatisfação com a imagem corporal encontradas neste estudo, verificou-se que os adolescentes insatisfeitos (tanto pelo excesso de peso quanto pela magreza) tiveram maiores chances de ideação e planejamento de suicídio. A literatura não tem apresentado consenso sobre as relações entre imagem corporal e pensamentos e comportamentos suicidas. Em pesquisas realizadas com a população adolescente, Crow et al. ${ }^{19}$ verificaram, em adolescentes dos Estados Unidos, que os que estavam insatisfeitos tinham maior chance de ideação e tentativa de suicídio; du Roscoät et al. ${ }^{20}$, ao investigarem apenas a tentativa de suicídio, encontraram maior chance do desfecho em adolescentes franceses do sexo feminino e masculino que se sentiam muito magros, um pouco acima do peso e muito acima do peso, e também nos adolescentes do sexo masculino que se sentiam apenas um pouco magros. Por sua vez, no estudo de Brausch e Gutierrez ${ }^{18}$, conduzido nos Estados Unidos, foi observado que a imagem corporal não teve efeito direto nos pensamentos e comportamentos suicidas.

As divergências nos resultados do presente estudo bem como nos dos demais citados podem ter ocorrido devido às diferentes medidas de avaliação da insatisfação com a imagem corporal utilizadas, bem como das características das amostras investigadas e dos locais de realização das pesquisas. Apesar disso, conforme a literatura aponta que os fatores de risco podem contribuir diretamente para os pensamentos e comportamentos suicidas, mas também indiretamente ao influenciar a suscetibilidade individual aos transtornos mentais ${ }^{1}$, acredita-se que a insatisfação com a imagem corporal possa ter um efeito direto na ideação, planejamento e tentativa de suicídio mas principalmente indireto, por predispor os indivíduos a diversos transtornos mentais ${ }^{33}$. No entanto, o delineamento transversal do presente estudo impossibilita inferir a direção das relações entre pensamentos e comportamentos suicidas e insatisfação com a imagem corporal.

De modo geral, entende-se que os resultados deste estudo precisam ser interpretados considerando-se inúmeros fatores que podem afetar as prevalências de pensamentos e comportamentos suicidas em pesquisas de autorrelato, entre eles a extensão em que os indivíduos desejam revelar essas informações ${ }^{1}$. O mesmo apontamento vale para as informações sobre a imagem corporal.

Como orientações, sugere-se que a escola, sendo um local de constante debate sobre a saúde, pode promover discussões sobre o tema durante as aulas, utilizando uma abordagem multidisciplinar e levando em consideração os motivos que levam os adolescentes ao envolvimento em pensamentos e comportamentos suicidas. Ademais, por ser um espaço que lida cotidianamente com a população adolescente, a escola, pode ser uma grande aliada no desenvolvimento de programas de prevenção e tratamento, tanto da insatisfação com a imagem corporal quanto dos pensamentos e comportamentos suicidas.

\section{CONCLUSÕES}

O sexo feminino apresentou maiores prevalências de pensamentos e comportamentos suicidas comparado ao sexo masculino. Independentemente do sexo, idade, IMC e maturação sexual, os adolescentes insatisfeitos com a imagem corporal (pelo excesso de peso e pela magreza) estiveram mais suscetíveis à ideação e ao planejamento do suicídio comparados aos adolescentes satisfeitos.

\section{CONTRIBUIÇÕES INDIVIDUAIS}

Gaia Salvador Claumann e André de Araújo Pinto Realizaram a análise e interpretação dos dados, redigiram o artigo e aprovaram a versão final a ser publicada. 
Diego Augusto Santos Silva e Andreia Pelegrini Participaram da concepção do estudo, realizaram a revisão crítica do conteúdo e aprovaram a versão final a ser publicada.

\section{CONFLITOS DE INTERESSE}

Todos os autores declaram não possuir potenciais conflitos de interesse.

\section{REFERÊNCIAS}

1. World Health Organization. Preventing suicide: a global imperative. Luxembourg: WHO Press; 2014

2. Ministério da Saúde. Secretaria de Vigilância em Saúde. Perfil epidemiológico das tentativas e óbitos por suicídio no Brasil e a rede de atenção à saúde. Boletim Epidemiológico. 2017;30.

3. Chau K, Kabuth B, Chau N. Gender and family disparities in suicide attempt and role of socioeconomic, school, and health-related difficulties in early adolescence. Biomed Res Int. 2014;2014.

4. Dell'Aglio DD, Braga LL. Suicidal behavior in adolescents from different contexts in south of Brazil. J Lat Am Stud. 2015;7(1):67-81.

5. Park S. Gender-specific factors of suicide ideation among adolescents in the Republic of Korea: a nationally representative population-based study. Arch Psychiatr Nurs. 2013;27(5):253-9.

6. Peltzer K, Pengpid S. Suicidal ideation and associated factors among school-going adolescents in Thailand. Int J Environ Res Public Health. 2012;9(2):462-73.

7. Franklin JC, Ribeiro JD, Fox KR, Bentley KH, Kleiman EM, Huang X, et al. Risk factors for suicidal thoughts and behaviors: A meta-analysis of 50 years of research. Psychol Bull. 2017;143(2):187-232.

8. Markey CN. Invited commentary: why body image is important to adolescent development. J Youth Adolesc. 2010;39(12):1387-91.

9. Choi E, Choi I. The associations between body dissatisfaction, body figure, self-esteem, and depressed mood in adolescents in the United States and Korea: A moderated mediation analysis. J Adolesc. 2016;53:249-59.

10. Duchesne AP, Dion J, Lalande D, Bégin C, Émond C, Lalande G, et al. Body dissatisfaction and psychological distress in adolescents: Is self-esteem a mediator? J Health Psychol. 2017;22(12):1563-9.

11. Flores-Cornejo F, Kamego-Tome M, Zapata-Pachas MA, Alvarado GF. Association between body image dissatisfaction and depressive symptoms in adolescents. Rev Bras Psiquiatr. 2017;39(4):316-22.

12. Ceballos-0spino GA, Suarez-Colorado Y, Suescún-Arregocés J, Gamarra-Vega LM, González KE, Sotelo-Manjarres AP. Ideación suicida, depresión y autoestima en adolescentes escolares de Santa Marta. Duazary. 2015;12(1):15-22.

13. Rodríguez MJC, Pérez RR, Valdez EA, Barreras ALG. Depression and suicidal behavior among high school students in Sonora. Salud Mental. 2012;35:39-44.
14. Shaikh MA, Lloyd J, Acquah E, Celedonia KL, L Wilson M. Suicide attempts and behavioral correlates among a nationally representative sample of school-attending adolescents in the Republic of Malawi. BMC Public Health. 2016;16(1):843.

15. Siabato Macías EF, Forero Mendoza IX, Salamanca Camargo Y. Asociación entre depresión e ideación suicida en un grupo de adolescentes colombianos. Pensam Psicol. 2017;15(1):51-61.

16. Muehlenkamp JJ, Brausch AM. Body image as a mediator of non-suicidal self-injury in adolescents. J Adolesc. 2012;35(1):1-9.

17. Duggan JM, Toste JR, Heath NL. An examination of the relationship between body image factors and non-suicidal self-injury in young adults: the mediating influence of emotion dysregulation. Psychiatry Res. 2013;206(2-3):256-64.

18. Brausch AM, Gutierrez PM. The role of body image and disordered eating as risk factors for depression and suicidal ideation in adolescents. Suicide Life Threat Behav. 2009;39(1):58-71.

19. Crow S, Eisenberg ME, Story M, Neumark-Sztainer D. Suicidal behavior in adolescents: relationship to weight status, weight control behaviors, and body dissatisfaction. Int J Eat Disord. 2008;41(1):82-7.

20. du Roscoät E, Legleye S, Guignard R, Husky M, Beck F. Risk factors for suicide attempts and hospitalizations in a sample of 39,542 French adolescents. J Affect Disord. 2016;190:517-21.

21. Silva DA, Tremblay M, Pelegrini A, Dos Santos Silva RJ, Cabral de Oliveira AC, Petroski EL. Association between aerobic fitness and high blood pressure in adolescents in Brazil: evidence for criterion-referenced cut-points. Pediatr Exerc Sci. 2016;28(2):312-20.

22. Guedes DP, Lopes CC. Validação da versão brasileira do Youth Risk Behavior Survey 2007. Rev Saúde Pública. 2010;44(5):840-50.

23. Stunkard AJ, Sørensen T, Schulsinger F. Use of the Danish Adoption Register for the study of obesity and thinness. Res Publ Assoc Res Nerv Ment Dis. 1983;60:115-20.

24. Scagliusi FB, Alvarenga M, Polacow V0, Cordás TA, de Oliveira Queiroz GK, Coelho D, et al. Concurrent and discriminant validity of the Stunkard's figure rating scale adapted into Portuguese. Appetite. 2006;47(1):77-82.

25. Stewart A, Marfell-Jones M, Olds T, Ridder, H. International Standards for Anthropometric Assessment - ISAK. New Zealand: Lower Hutt; 2011

26. Adami F, Vasconcelos FAG. Obesidade e maturação sexual precoce em escolares de Florianópolis - SC. Rev Bras Epidemiol. 2008;11(4):549-60.

27. Zeller MH, Reiter-Purtill J, Jenkins TM, Ratcliff MB. Adolescent suicidal behavior across the excess weight status spectrum. Obesity (Silver Spring). 2013;21(5):1039-45.

28. Arnarsson A, Sveinbjornsdottir S, Thorsteinsson EB, Bjarnason T. Suicidal risk and sexual orientation in adolescence: a population-based study in Iceland. Scand J Public Health. 2015;43(5):497-505

29. Sharma B, Nam EW, Kim HY, Kim JK. Factors Associated with Suicidal Ideation and Suicide Attempt among School-Going Urban Adolescents in Peru. Int J Environ Res Public Health. 2015;12(11):14842-56

30. Souza LDM, Ores L, Oliveira GT, Cruzeiro ALS, Silva RA, Pinheiro RT, et al. Ideação suicida na adolescência: prevalência e fatores associados. J Bras Psiquiatr. 2010;59(4):286-92.

31. Silva RJS, Santos FAL, Soares NMM, Pardono E. Suicidal ideation and associated factors among adolescents in Northeastern Brazil. Sci World J. 2014;2014:8.

32. Veras $J L$, Ximenes RC, de Vasconcelos FM, Sougey EB. Prevalence of suicide risk among adolescents with depressive symptoms. Arch Psychiatr Nurs. 2016;30(1):2-6.

33. Bucchianeri MM, Neumark-Sztainer D. Body dissatisfaction: an overlooked public health concern. J Public Ment Health. 2014;13(2):64-9. 